\title{
Limits of Local Search: Quality and Efficiency
}

\author{
Norbert Bus \\ Université Paris-Est, \\ Laboratoire d'Informatique Gaspard-Monge, \\ Equipe A3SI, ESIEE Paris. \\ busn@esiee.fr \\ Shashwat Garg \\ IIT Delhi \\ garg.shashwat@gmail.com
}

\author{
Nabil H. Mustafa \\ Université Paris-Est, \\ Laboratoire d'Informatique Gaspard-Monge, \\ Equipe A3SI, ESIEE Paris. \\ mustafan@esiee.fr \\ Saurabh Ray \\ Computer Science, New York University, Abu Dhabi. \\ saurabh.ray@nyu.edu
}

\begin{abstract}
Over the past several decades there has been steady progress towards the goal of polynomial-time approximation schemes (PTAS) for fundamental geometric combinatorial optimization problems. A foremost example is the geometric hitting set problem: given a set $P$ of points and a set $\mathcal{D}$ of geometric objects, compute the minimum-sized subset of $P$ that hits all objects in $\mathcal{D}$. For the case where $\mathcal{D}$ is a set of disks in the plane, the 30-year quest for a PTAS, starting from the seminal work of Hochbaum [22], was finally achieved in 2010 [26]. Surprisingly, the algorithm to achieve the PTAS is simple: local-search. In particular, the algorithm starts with any hitting set, and iteratively tries to decrease its size by trying to replace some $k$ points by $k-1$ points; call such an algorithm a $(k, k-1)$-local search algorithm.

Since then, local-search has turned out to be a powerful algorithmic approach towards achieving good approximation ratios for geometric problems (for geometric independent-set problem, for dominating sets, for the terrain guarding problem and several others). Unfortunately all these algorithms have the same limitation: local search is able to give a PTAS, but with large running times. In particular, the current best work shows that if $k \geq 30$, then local-search is able to give a constant factor (as a function of $k$ ) approximation ratio [17]. Unfortunately this then implies that the running time for local-search to provably work at all is $\Omega\left(n^{30}\right)$ using the current framework.

As currently local search is the only known method that gives approximation factors that could be useful in practice, it becomes important to explore the limits - in both efficiency and quality - of local search. Simple examples show that $(1,0)$ and $(2,1)$ local search cannot give constant factor approximations. In this paper, we show that, surprisingly, just $(3,2)$ local search is able to give a constant-factor approximation; in fact we are able to get the precise quality limit of $(3,2)$-local search: factor 8 approximation. This simplest working instance of local search already gives an approximation factor that is better than all known other methods! In fact, our improvement applies to all algorithms that use local-search for geometric independentset problem, for dominating sets, for the terrain guarding problem and several others. Finding efficient
\end{abstract}


$(3,2)$-local search algorithms then becomes the key bottleneck in efficient and good-quality algorithms. In this paper we present such improved algorithms.

\section{Introduction}

The minimum hitting set problem is one the most fundamental combinatorial optimization problems: given a range space $(P, \mathcal{D})$ consisting of a set $P$ and a set $\mathcal{D}$ of subsets of $P$ called the ranges, the task is to compute the smallest subset $S \subseteq P$ that has a non-empty intersection with each of the ranges in $\mathcal{D}$. This problem is strongly NP-hard. If there are no restrictions on the set system $\mathcal{D}$, then it is known that it is NP-hard to approximate the minimum hitting set within a logarithmic factor of the optimal [30]. A natural occurrence of the hitting set problem occurs when the range space $\mathcal{D}$ is derived from geometry. For example, given a set $P$ of $n$ points in $\mathbb{R}^{2}$, and a set $\mathcal{D}$ of $m$ triangles containing points of $P$, compute the minimum-sized subset of $P$ that hits all the triangles in $\mathcal{D}$. Unfortunately, for most natural geometric range spaces, computing the minimum-sized hitting set remains NP-hard. For example, even the (relatively) simple case where $\mathcal{D}$ is a set of unit disks in the plane is strongly NP-hard [21]. Therefore fast algorithms for computing provably good approximate hitting sets for geometric range spaces have been intensively studied for the past three decades (e.g., see the two recent $\mathrm{PhD}$ theses on this topic $[17,18])$. Since there is little hope of computing the minimum-sized hitting set for general geometric problems in polynomial time, effort has turned to approximating the optimal solution.

In this paper we will consider a fundamental case for geometric hitting sets, where the geometric objects are arbitrary radius disks in the plane (or halfspaces in $\mathbb{R}^{3}$ ). This has been the subject of a long line of investigation, for more than two decades. The case when all the disks have the same radius is easier, and has been studied in a series of works: Călinsecu et al. [10] proposed a 108-approximation algorithm, which was subsequently improved by Ambhul et al. [6] to 72. Carmi et al. [11] further improved that to a 38-approximation algorithm, though with the running time of $O\left(n^{6}\right)$. Claude et al. [13] were able to achieve a 22-approximation algorithm running in time $O\left(n^{6}\right)$. More recently Fraser et al. [19] presented a 18-approximation algorithm in time $O\left(n^{2}\right)$.

The problem becomes harder when the disk radii can be arbitrary. A first break-through for this problem came in 1994, when Bronnimann and Goodrich [8] proved the following interesting connection between the hittingset problem, and $\epsilon$-nets ${ }^{1}$ : let $(P, \mathcal{D})$ be a range-space for which we want to compute a minimum hitting set. If one can compute an $\epsilon$-net of size $c / \epsilon$ for the $\epsilon$-net problem for $(P, \mathcal{D})$ in polynomial time, then one can compute a hitting set of size at most $c \cdot$ OPT for $(P, \mathcal{D})$, where OPT is the size of the optimal (smallest) hitting set, in polynomial time. A shorter, simpler proof was given by Even et al. [15]. Recently, Agarwal-Pan [5] presented an algorithm that can compute hitting-sets for disks from $\epsilon$-nets in time $O\left(n \log ^{6} n\right)$, improving on the previous near-linear time algorithm which guaranteed a $O(\log n)$-approximation [3].

\subsection{Local search.}

There is a fundamental limitation of the above technique: it cannot give better than constant-factor approximations. The reason is that the technique reduces the problem of computing a minimum size hitting set to the problem of computing a minimum sized $\epsilon$-net. And it is known that for some constant $c \geq 2$, there do not exist

\footnotetext{
${ }^{1}$ Given a range space $(P, \mathcal{D})$, an $\epsilon$-net is a subset $S \subseteq P$ such that $D \cap S \neq \emptyset$ for all $D \in \mathcal{D}$ with $|D \cap P| \geq \epsilon n$.
} 


\begin{tabular}{|l|c|c|}
\hline \multicolumn{3}{|c|}{ CONGRUENT DISKS } \\
\hline & Quality & Time \\
\hline Călinsecu et al. [10] & 108 & $O\left(n^{2}\right)$ \\
\hline Ambhul et al. [6] & 72 & $O\left(n^{2}\right)$ \\
\hline Carmi et al. [11] & 38 & $O\left(n^{6}\right)$ \\
\hline Claude et al. [13] & 22 & $O\left(n^{6}\right)$ \\
\hline Fraser et al. [19] & 18 & $O\left(n^{2}\right)$ \\
\hline Acharyya et al. [1] & $(9+\epsilon)$ & $O\left(n^{2(1+6 / \epsilon)+1)}\right)$ \\
\hline
\end{tabular}

\begin{tabular}{|l|c|c|}
\hline \multicolumn{3}{|c|}{ ARBITRARY DISKS } \\
\hline & Quality & Time \\
\hline Bronniman et al. [8] & $O(1)$ & $O\left(n^{3}\right)$ \\
\hline Mustafa et al. [26] & $(1+\epsilon)$ & $n^{O\left(1 / \epsilon^{2}\right)}$ \\
\hline Agarwal et al. [3] & $O(\log n)$ & $\tilde{O}(n)$ \\
\hline Agarwal et al. [5] & $O(1)$ & $\tilde{O}(n)$ \\
\hline This paper & $8+\epsilon$ & $\tilde{O}\left(n^{7 / 3}\right)$ \\
\hline
\end{tabular}

Table 1: Summary of previous work.

$\epsilon$-nets of size smaller than $c / \epsilon$ - even for halfspaces in 2D. This limitation was the main barrier towards better quality algorithms until the usefulness of local search algorithms was introduced.

There has been recent progress in breaking the constant-approximation barriers for many geometric problems using very similar applications of local-search; e.g., dominating sets in disk intersection graphs [20], terrain guarding problem [23], independent set of pseudodisks [12] and several other problems. For the hitting set problem on $(P, \mathcal{D})$, local-search works as follows: start with any hitting set $S \subseteq P$, and repeatedly decrease the size of $S$, if possible, by replacing $k$ points of $S$ with $<k$ points of $P \backslash S$. Call such an algorithm a $(k, k-1)$-local search algorithm. Mustafa-Ray [26] showed that for a large-enough value of $k$, a $(k, k-1)$ local search algorithm for the hitting set problem gives a $(1+c / \sqrt{k})$-approximation, for a fixed constant $c$, when the ranges are disks, or more generally, pseudo-disks in $\mathbb{R}^{2}$. The running time of their algorithm to compute a $(1+\epsilon)$-approximation is $O\left(n^{O\left(1 / \epsilon^{2}\right)}\right)$.

\subsection{Our Contributions.}

Both these approaches have to be evaluated on the questions of computational efficiency as well as approximation quality. In spite of all the progress, there remains a large gap between quality and efficiency - mainly due to the ugly trade-offs between running times and approximation factors; see Table 1 for the current state of the art. The algorithm of Agarwal-Pan [5] that rounds via $\epsilon$-nets gives an $\tilde{O}(n)$-time algorithm, but the constant in the approximation depends on the constant in the size of $\epsilon$-nets, which is large. At the other end, the $(k, k-1)$-local search algorithm [26] can compute solutions arbitrarily close to the optimal, but it is extremely inefficient, even for reasonable approximation factors. In particular, the current best work shows that if $k \geq 30$, then local-search is able to give a constant factor (as a function of $k$ ) approximation ratio [17]. Unfortunately this then implies that the running time for local-search to provably work at all is $\Omega\left(n^{30}\right)$ using the current framework. Furthermore, note that any attempts at progress on improving local search must take into account that the hitting set problem for even unit disks is $W[1]$-hard [24]; so it is unlikely that algorithms exist that do not have a dependency on $1 / \epsilon$ in the exponent.

As currently local search is the only known method that gives approximation factors that could be useful in practice, it becomes important to explore the limits - in both efficiency and quality - of local search. Simple examples show that $(1,0)$ and $(2,1)$ local search cannot give constant factor approximations. In this paper, we 
show that, surprisingly, just $(3,2)$ local search is able to give a constant-factor approximation; in fact we are able to get the precise quality limit of $(3,2)$-local search: factor 8 approximation. This simplest working instance of local search already gives an approximation factor that is better than all known other methods! Finding efficient $(3,2)$-local search algorithms then becomes the key concern in efficient and good-quality algorithms. In this paper we present such improved algorithms. Table 1 states our contributions.

A straightforward algorithm for $(3,2)$-local search proceeds as follows: each $(3,2)$ improvement step tries all $O\left(n^{5}\right)$ 5-tuples, and for each checks if it is indeed an improvement in time $O(n)$. The total number of steps for the whole algorithm can be $O(n)$, giving a $O\left(n^{7}\right)$ naive running time. Our main result is to show how to perform this search much more efficiently:

Theorem (Proof in Section 2). A (3,2)-local search can be performed in expected time $O\left(n^{2.34}\right)$.

We then determine the exact limits of $(3,2)$-local search:

Theorem (Proof in Section 3). A (3,2)-local search algorithm returns a 8-approximation to the minimum hitting set. Furthermore, there exist a set $P$ of points and a set $\mathcal{D}$ of disks where $(3,2)$ local-search does not return hitting-sets of size less than 8 factor of the optimal hitting set.

Remark: In fact this immediately implies improved bounds for many other local search algorithms; e.g., it implies that the $(3,2)$-local search gives 8 -approximation to the independent set of pseudodisks, dominating sets in disk intersection graphs, terrain guarding problem.

We also prove improved approximation bounds resulting from $(4,3)$-local search:

Theorem (Proof in Section 3). A (4,3)-local search algorithm returns a 5-approximation to the minimum hitting set problem.

\section{Analysis of Efficiency of Local Search}

Our algorithm is based on local search. It starts with a hitting set and repeatedly tries to make local improvements. Let $S$ be a hitting set for $\mathcal{H}(P, \mathcal{D})$. Let $X \subseteq S$ and $Y \subseteq P$. We say that $(X, Y)$ is a local improvement pair with respect to $S$ and $\mathcal{H}(P, \mathcal{D})$ if $|Y|<|X|$ and $(S \backslash X) \cup Y$ is a hitting set for $\mathcal{H}(P, \mathcal{D})$. Such a local improvement reduces the size of the hitting set by $|X|-|Y|$. We will refer to this quantity as the profit of the local improvement and the local improvement pair. We say that $X \subseteq S$ is locally improvable with respect to $S$ and $\mathcal{H}(P, \mathcal{D})$ if there exists a $Y \subseteq P$ such that $(X, Y)$ is a local improvement pair. If $(X, Y)$ is a local improvement pair, we say that $Y$ can locally replace $X$.

Let $S$ be a hitting set for $\mathcal{H}(P, \mathcal{D})$. For any $s \in S$, we denote by $\mathcal{D}(s)$ the set of disks in $\mathcal{D}$ that are hit by $s$ but not by any other point in $S$. We will call the disks in $\mathcal{D}(s)$ the personal disks of $s$. We will denote the region $\bigcap_{D \in \mathcal{D}(s)} D$ by $R(s)$ and call it the personal region of $s$. The notations $\mathcal{D}(s)$ and $R(s)$ are always with respect to a set system $\mathcal{H}(P, \mathcal{D})$ and a hitting set $S$. These things that are not explicit in the notation will be clear from the context. We also extend the same definitions for sets of points: for a set $X \subseteq S$, let $\mathcal{D}(X)$ be the set of disks in $\mathcal{D}$ which contain only points of $X$. We call these the personal disks of $X$. The personal region of $X$ is 
$R(X)=\bigcap_{D \in \mathcal{D}(X)} D$. A set of regions $\mathcal{R}$ are pseudodisks if they are simply connected and the boundaries of every pair of regions in $\mathcal{R}$ intersect at most twice.

\section{Preparing for the algorithm.}

Before presenting our algorithm, we prove a few results useful for describing the algorithm.

Lemma 1. Let $S$ be a hitting set for $\mathcal{H}(P, \mathcal{D})$. If $|S|>8 \cdot \operatorname{OPT}(P, \mathcal{D})+3 t$, for some integer $t \geq 0$, then there exist $t+1$ disjoint subsets $X_{0}, \ldots, X_{t}$ of $S$, each of which is of size 3 and is locally improvable with respect to $S$ in $\mathcal{H}(P, \mathcal{D})$.

Proof. The proof is by induction. The statement is true for $t=0$ : if there is no locally improvable set $X$ of size 3 , then taking $B=S$ and $R=O$, where $O$ is the optimal hitting set for $\mathcal{H}(P, \mathcal{D})$ and applying Theorem 2, we get that $|S| \leq 8 \operatorname{Opt}(P, \mathcal{D})$. Assume inductively that the lemma is true for $t-1$, and let the $t$ disjoint sets of $S$ be $X_{0}, \cdots, X_{t-1}$. It remains to construct the set $X_{t}$. Let $Z=\bigcup_{i=0}^{t-1} X_{i}$. Let $P^{\prime}=P \backslash Z$, $\mathcal{D}^{\prime}=\{D \in \mathcal{D}: D \cap Z=\emptyset\}$ and $S^{\prime}=S \backslash Z$. Clearly $S^{\prime}$ is a hitting set for $\mathcal{H}\left(P^{\prime}, \mathcal{D}^{\prime}\right)$. Moreover,

Claim 2.1. $\operatorname{OpT}\left(P^{\prime}, \mathcal{D}^{\prime}\right) \leq \operatorname{Opt}(P, \mathcal{D})$.

Proof. Take any hitting set $A$ for $\mathcal{H}(P, \mathcal{D})$. Then any point $a \in A$ that hits a disk in $\mathcal{D}^{\prime}$ must belong to $P^{\prime}$ : otherwise $a \in P \backslash P^{\prime}=Z$, and we had constructed $\mathcal{D}^{\prime}$ by removing all the disks hit by $Z$ from $\mathcal{D}$. Therefore all the points in $A$ hitting $\mathcal{D}^{\prime}$ belong to $P^{\prime}$, and form a hitting set in $P^{\prime}$ for $\mathcal{H}\left(P^{\prime}, \mathcal{D}^{\prime}\right)$ of size at most $|A|$.

Therefore, $\left|S^{\prime}\right|=|S|-3 t>8 \cdot \operatorname{OpT}(P, \mathcal{D}) \geq 8 \cdot \operatorname{Opt}\left(P^{\prime}, \mathcal{D}^{\prime}\right)$, and any hitting set for $\mathcal{H}(P, \mathcal{D})$ contains a hitting set for $\mathcal{H}\left(P^{\prime}, \mathcal{D}^{\prime}\right)$. Now, using the Theorem 2 for $t=0$ on $S^{\prime}$ and $\left(P^{\prime}, \mathcal{D}^{\prime}\right)$, the fact that $\left|S^{\prime}\right|>8 \cdot \operatorname{Opt}\left(P^{\prime}, \mathcal{D}^{\prime}\right)$ implies that there is set $X_{t} \subseteq S^{\prime}$ of size 3 and a set $Y \subseteq P^{\prime}$ of size 2 such that $\left(S^{\prime} \backslash X_{t}\right) \cup Y$ is a hitting set for $\mathcal{H}\left(P^{\prime}, \mathcal{D}^{\prime}\right)$. This means that $\left(S^{\prime} \backslash X_{t}\right) \cup Y \cup Z$ is a hitting set for $\mathcal{H}(P, \mathcal{D})$ since all disks in $\mathcal{D} \backslash \mathcal{D}^{\prime}$ intersect $Z$. In other words, $\left(S \backslash X_{t}\right) \cup Y$ is a hitting set for $\mathcal{H}(P, \mathcal{D})$ since $S^{\prime} \cup Z=S$ and $X_{t} \cap Z=\emptyset$. That is, $X_{t}$ is locally improvable with respect to $S$ in $\mathcal{H}(P, \mathcal{D})$. Since $X_{t} \subseteq S^{\prime}$ and $S^{\prime} \cap Z=\emptyset, X_{t}$ is disjoint from the other $X_{i}$ 's.

The following key structural property is crucial:

Lemma 2. Let $S$ be a hitting set for $\mathcal{H}(P, \mathcal{D})$. Then the personal regions of the points in $S$ form a collection of pseudodisks.

Proof. First observe that since each personal region $R(s)$ is an intersection of disks, it is convex. We show that for any two points $x, y \in S, R(x)$ and $R(y)$ are non-piercing i.e., the regions $R(x) \backslash R(y)$ and $R(y) \backslash R(x)$ are connected. Since the boundaries of two convex regions that are non-piercing cannot intersect in more than two points, we conclude that the regions form a collection of pseudodisks.

Assume for contradiction that $R(x)$ and $R(y)$ are piercing and without loss of generality that $R(x) \backslash R(y)$ has at least two connected components. The point $x$ lies in one of these components and let $x^{\prime}$ be a point in a different component of $R(x) \backslash R(y)$. Since $x^{\prime}$ does not lie in $R(y)$, there must be a disk $D_{y} \in \mathcal{D}(y)$ that does not contain $x^{\prime}$. Since no disk in $\mathcal{D}(y)$ contains $x, D_{y}$ also does not contain $x$. Since $D_{y}$ contains $R(y)$, this implies that $x$ 


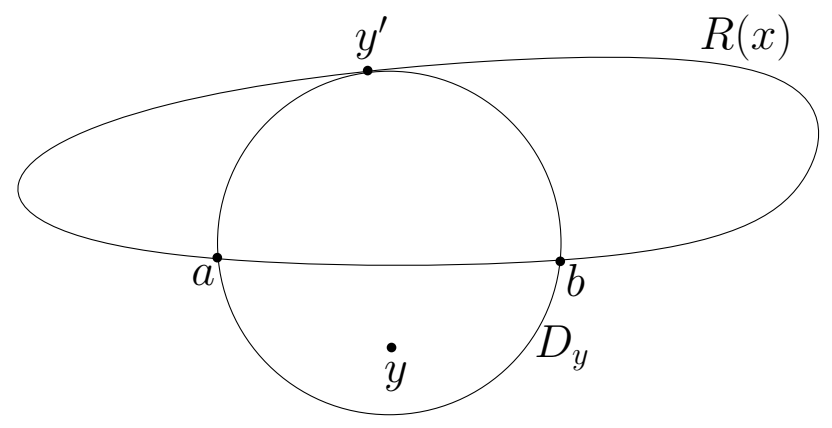

Figure 1: Case 2 in the proof of Lemma 2.

and $x^{\prime}$ are in different connected components of $R(x) \backslash D_{y}$. In other words $R(x) \backslash D_{y}$ is not connected. There are two cases to consider now:

Case 1: $D_{y} \backslash R(x)$ is not connected. In this case $y$ lies in one of the connected components of $D \backslash R(x)$. Let $y^{\prime}$ be a point in one of the other components. Since $y^{\prime} \notin R(x)$, there is some disk $D_{x} \in \mathcal{D}(x)$ which does not contain $y^{\prime}$ and by assumption does not contain $y$. Since $D_{x}$ contains $R(x)$, this implies that $D_{y} \backslash D_{x}$ is not connected, a contradiction as $D_{x}, D_{y}$ are disks.

Case 2: $D_{y} \backslash R(x)$ is connected. In this case, since $R(x) \backslash D_{y}$ is not connected, the situation is as shown in Figure 1. There is at least one point $y^{\prime}$ where the boundaries of $D_{y}$ and $R(x)$ intersect but do not cross. Furthermore, $y^{\prime}$ does not lie on the boundary of $D_{y} \backslash R_{x}$. Since $y^{\prime}$ lies on the boundary of $R_{x}$, it lies on the boundary of some disk $D_{x} \in \mathcal{D}(x)$. Note that $D_{x}$ contains $R_{x}$ but does not contain $y$. Therefore, $D_{x}$ must intersect the boundary of $D_{y}$ at least twice in the portion of $\partial D_{y}$ that lies outside $R_{x}$, i.e., the arc between $a$ and $b$ (shown in the figure) in counterclockwise direction. The boundaries of $D_{x}$ and $D_{y}$ then intersect at least three times, a contradiction for disks.

Lemma 3. Let $S$ be a hitting set for $\mathcal{H}(P, \mathcal{D})$. Suppose that we are given two sets $X \subseteq S$ and $Y \subseteq P$ such that $|Y|=O(1),|X|>4|Y|$ and for each $x \in X, Y$ hits $\mathcal{D}(x)$, the personal disks of $x$. Then there exists a set $X^{\prime} \subseteq X$ of size $\Omega(|X|)$ such that $\left(X^{\prime}, Y\right)$ is a local improvement pair with respect to $S$ and $\mathcal{H}(P, \mathcal{D})$. Furthermore, given $X$ and $Y, X^{\prime}$ can be computed in time $O(|X| \log |X|)$.

Proof. Consider the Delaunay triangulation of the points in $X$, and let $X^{\prime}$ be an independent-set in this Delaunay graph. First we show that $\left(S \backslash X^{\prime}\right) \cup Y$ is a hitting set for $\mathcal{H}(P, \mathcal{D})$. Consider a disk $D$ that is not hit by $S \backslash X^{\prime}$. Since $D$ is hit by $S\left(S\right.$ being a hitting set for $\mathcal{H}(P, \mathcal{D})$ ), $D$ contains at least one point of $X^{\prime}$. If $D$ contains exactly one point $x^{\prime} \in X^{\prime}$ then $D$ is hit by $Y$ since $D \in \mathcal{D}\left(x^{\prime}\right)$ and $Y$ hits $\mathcal{D}\left(x^{\prime}\right)$. Otherwise, $D$ contains at least two points of $X^{\prime}$, in which case it must contain some point of $x \in X \backslash X^{\prime} \subseteq S \backslash X^{\prime}$ since $X^{\prime}$ is an independent set in the Delaunay triangulation of $X$ (and by the fact that subgraph of the Delaunay graph induced by the set of points of $X$ inside any disk is connected).

The Delaunay triangulation can be constructed in $O(|X| \log |X|)$ time. If $|X| \leq 5|Y|$, i.e. $|X|=O(1)$, we find an independent set of size at least $\lceil|X| / 4\rceil>|Y|$ in the Delaunay graph in $O(1)$ time by brute force; 
the existence of such an independent set follows from the 4-color theorem on planar graphs. If $|X|>5|Y|$, we compute a 5 -coloring of the Delaunay graph in $O(|X|)$ time and take the largest color class as $X^{\prime}$. Thus $\left|X^{\prime}\right| \geq\lceil|X| / 5\rceil>|Y|$.

Therefore $\left|X^{\prime}\right|>|Y|$, and so $\left(X^{\prime}, Y\right)$ is a local improvement pair.

The next two lemmas show that one can efficiently preprocess disks to answer containment queries in logarithmic time.

Lemma 4. Let $\mathcal{D}$ be a set of $m$ disks in the plane having a common intersection region, say $R$. Then the boundary of $R$ is composed of $O(m)$ circular arcs, and can be computed in $O(m \log m)$ expected time. We can also construct, in $O(m \log m)$ time, a data structure which, for any given query point $q$, answers whether $q \in R$ in $O(\log m)$ time.

Proof. Let $p$ be a point in the region $R$ and $D$ be disk in $\mathcal{D}$. Consider the function $f_{D}(\theta)$, for $\theta \in[0,2 \pi)$, to be the distance from $p$ to the boundary of $D$ in the direction $\theta$ (i.e., along a ray emanating from $p$ at an angle $\theta$ to the positive $x$ axis). Clearly the graph of $f_{D}(\theta)$ with $\theta$ plotted along the $x$-axis is an $x$-monotone curve which we denote by $\Gamma_{D}$. Furthermore, for any two disks $D, D^{\prime} \in \mathcal{D}$ the curves $\Gamma_{D}$ and $\Gamma_{D^{\prime}}$ intersect at most twice since the boundaries of any two disks intersect at most twice. The curves in $\left\{\Gamma_{D}: D \in \mathcal{D}\right\}$ therefore form a set pseudo-parabolas. The number of arcs in the boundary of $R$ is equal to the size of the lower envelope of the arrangement of curves, which is $O(m)$ due to the linear union complexity of pseudo-parabolas [28, 4].

The boundary of $R$ can be computed by a randomized incremental construction in $O(m \log m)$ in the same way as the union of a set of $m$ regions with linear union complexity is computed in the same amount of time. There is also a deterministic algorithm which takes $O\left(m \log ^{2} m\right)$ time. It basically splits the disks in $\mathcal{D}$ into disjoint sets of half the size, computes the intersection region for each, and then takes the intersection of the two intersection regions - which can be computed using a circular sweep. For details see $[27,16,4]$ and the references therein.

Once $R$ has been computed, we can easily set up a data structure that checks for a query point $q$ whether $q \in R$. The region $R$ is almost like a convex polygon except that its sides are circular arcs. We can take one vertex $v$ of the region $R$ and join it with a chord to each of the other vertices. The chords define a linear order. Given a query point $q$, we can determine, by binary search, two consecutive chords so that the cone defined by $v$ and the rays emanating from $v$ along the two chords contains $q$. Next we just need to check whether $q$ lies on the same side of the circular arc bounding $R$ in that cone, as $v$. Setting up this data structure takes $O(m)$ time and the query time is $O(\log m)$ as required.

Lemma 5. Let $P$ be a set of $n$ points in the plane and let $\mathcal{D}$ be a set of pseudodisks, the boundary of each being composed of circular arcs. For any constant $C$, we can compute, for each $p \in P$ that lies in at most $C$ pseudodisks, the exact set of pseudodisks it hits in $O(n \log m)$ time, where $m$ is the total number of arcs in all the pseudodisks.

Proof. We first compute the shallow levels (of depth at most $C$ ) of the arrangement of pseudodisks $\mathcal{D}$. This can be done using a randomized incremental construction in exactly the same way as the union of a set of pseudodisks is computed. This takes $O(m \log m)$ time since the overall complexity of the shallow levels (for 
constant $C$ ) is linear. For details see [27, 16, 4]. Once the shallow levels are computed, we set up a point location data structure. Then in $O(\log m)$ time per point, we can determine for each point exactly which of the at most $C$ pseudodisks it is contained in.

\section{The Algorithm.}

We now describe our algorithm for computing a small hitting set for $\mathcal{H}(P, \mathcal{D})$. We first compute a hitting set $S$ of size $O(\mathrm{OPT})$. This can be done using the near linear time algorithm of Agarwal-Pan [5]. We also assume that we know the value of $\mathrm{OPT}=\operatorname{Opt}(P, D)$ although it suffices to guess the value of OPT within a $(1+\epsilon)$ factor which can be done in $O(1 / \log (1+\epsilon))$ guesses since we know OPT within a constant factor. Throughout this section, we will use $n$ as the total input size. We therefore upper bound $|P|$ and $|D|$ by $n$.

Next, for a given $\epsilon>0$, we prune the input so that no point is contained in more than $\Delta=n /(\epsilon \cdot$ OpT $)$ disks. This can be done by iterating over each point $p \in P$ and computing the number of disks $\mathcal{D}^{\prime} \subseteq \mathcal{D}$ that contain $p$. If $\left|\mathcal{D}^{\prime}\right| \geq \Delta$, remove the disks in $\mathcal{D}^{\prime}$ from $\mathcal{D}$ and add the point $p$ to the set $Q$ (which is initially empty). Note that as we go over the points the set $\mathcal{D}$ changes but we do not change the value of $\Delta$. Since each time we add a point to $Q$, we remove at least $\Delta$ disks from $\mathcal{D},|Q| \leq n / \Delta=\epsilon$. OpT. We can thus add the set $Q$ to our hitting set at the cost of an added $\epsilon$ in our approximation factor. This preprocessing procedure takes $O\left(n^{2}\right)$ time (this will not be the bottleneck of our algorithm).

After preprocessing, we pass $P$ and $\mathcal{D}$ to Algorithm 1 which we describe now. It requires an initial hitting set $S$ of size $O($ OPT $)$ which we obtain from [5]. The goal of Algorithm 1 is to compute a hitting set whose size is at most $(8+\epsilon) \cdot$ OPT. We compute a value $t=|S|-8 \cdot$ OPT which indicates how far we are from the solution we seek. As we will see, when $t$ is large, progress can be made quickly. However as we approach the quantity 8 - OPT, progress becomes slower and slower. The algorithm uses only local improvements of the type $(X, Y)$ where $|Y| \leq 2$. Throughout the algorithm we maintain for each $D \in \mathcal{D}$, the number of points, $N_{D}$, it contains from $S$. Initially computing $N_{D}$ for each disk takes $O\left(n^{2}\right)$ time. After that we need to update these quantities only when a local improvement $(X, Y)$ happens. We update $N_{D}$ as follows: $N_{D}=N_{D}-|D \cap X|+|D \cap Y|$. Since $|Y|$ is always at most 2 in our algorithm, naively this takes time $O(n|X|)$ for updating all disks in $\mathcal{D}$. Since such a local improvement decreases the size of the hitting set $S$ by $|X|-2=\Omega(|X|)$, the overhead for maintaining $N_{D}$ is $O(n)$ per improvement. Let Locally Improve $(X, Y)$ be the procedure that updates $S$ to $(S \backslash X) \cup Y$ and updates $N_{D}$ for each disk as mentioned above.

In each iteration of the while loop in Algorithm 1, we first construct a range reporting data structure [2] for the points in $S$ so that given any disk $D$, we can find the set of points in $D \cap S$ in time $O(\log n+|D \cap S|)$. We then use this data structure to compute the personal disks of each $s \in S$ as follows. Iterate over each disk $D \in \mathcal{D}$ and if $N_{D}=1$, use the reporting data structure to find the single point $s \in S$ that is contained by $D$. We then add $D$ to the (initially empty) list of personal disks of $s$. Since each query takes $O(\log n)$ time, the total time taken to compute the personal disks is $O(n \log n)$. If we find some point $s \in S$ for which $\mathcal{D}(s)=\emptyset$, we can just remove $s$ from the current hitting set. In other words we do a local improvement $(\{s\}, \emptyset)$.

The algorithm iterates over the points in $P$ in random order, considering the possibility of replacing each point in a local-improvement step. Say the current point being considered is $p_{1}$; the goal is to find a point $p_{2}$ so that $\left\{p_{1}, p_{2}\right\}$ can replace a large set $X \subseteq S$, i.e., a local improvement pair $\left(X,\left\{p_{1}, p_{2}\right\}\right)$ of large profit. If we can find such a profitable local improvement, we make the improvement, exit from the for loop, and continue with 


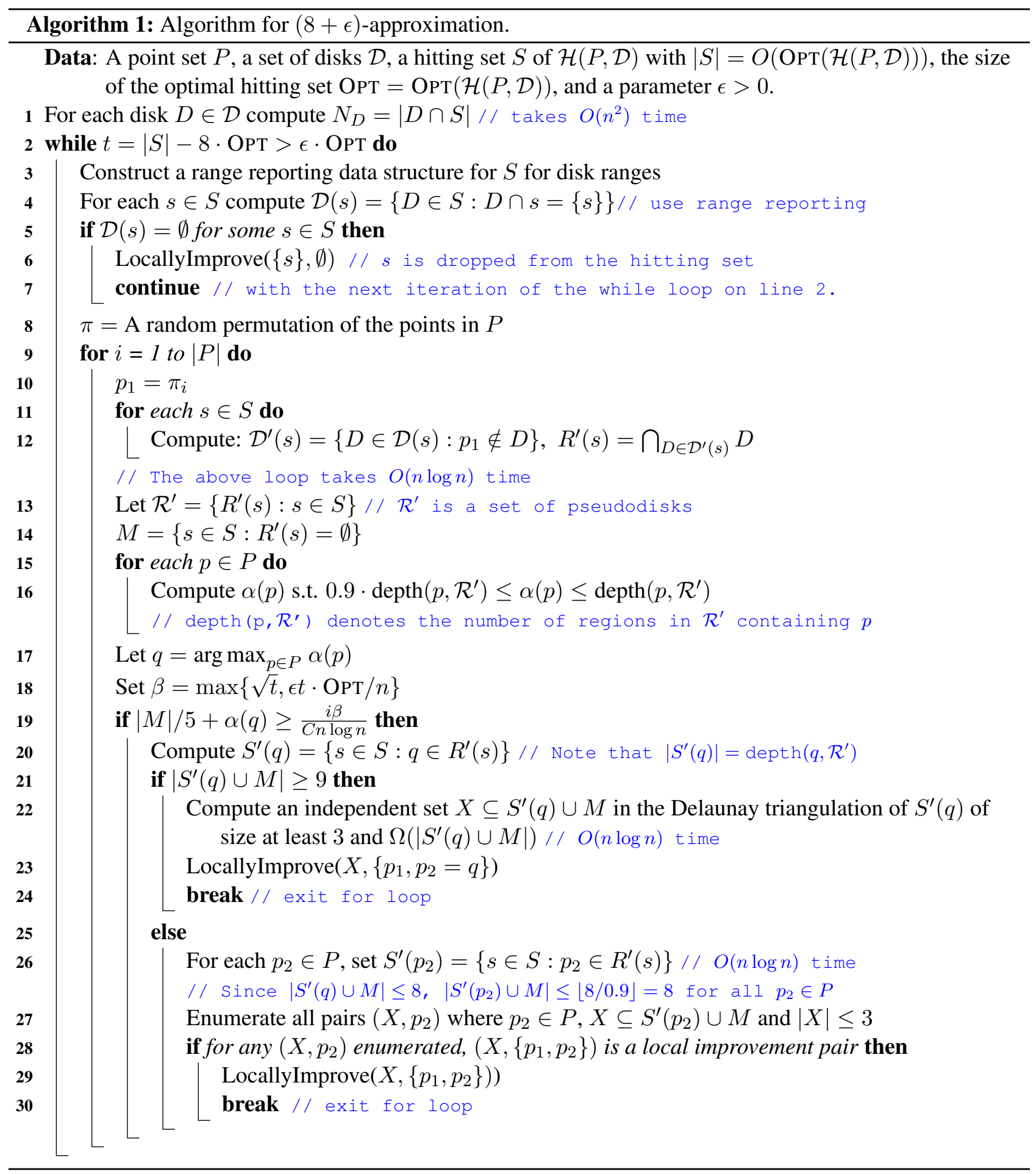


the next iteration of the while loop. Otherwise, we continue with the next point in the random ordering. For any pair of points $Y=\{p, q\} \subseteq P$, denote by $\rho(Y)$ the number of points in $S$ all of whose personal disks are hit by $Y$. For a point $p \in P$, we use $\rho(p)$ to denote $\max _{q \in P \backslash S} \rho(\{p, q\})$. Call a point $p \in P$ useful if there exists some $q \in P$ so that for some $X \subseteq S,(X,\{p, q\})$ is a local improvement pair.

\section{Analysis of the Algorithm.}

Lemma 6. If $p_{1}$ is useful, we can compute in $O\left(n \log ^{2} n\right)$ time a local improvement of profit $\Omega\left(\rho\left(p_{1}\right)\right)$.

Proof. Let us start by considering how we could compute $\rho\left(p_{1}\right)$. In order to compute $\rho\left(p_{1}\right)$, we need to find a point $q$ so that the number of points $s \in S$ whose personal disks are hit by $\left\{p_{1}, q\right\}$ is maximized. To do this, we first compute for each $s \in S$, the set $\mathcal{D}^{\prime}(s)$ of disks in $\mathcal{D}(s)$ that are not hit by $p_{1}$. For each $s \in S$, we then construct the region $R^{\prime}(s)$ by taking the intersection of the disks in $\mathcal{D}^{\prime}(s)$. Let $\mathcal{R}^{\prime}=\left\{R^{\prime}(s): s \in S\right\}$. For some $s \in S, \mathcal{D}^{\prime}(s)$ may be empty and consequently some of the regions in $\mathcal{R}^{\prime}$ are empty. Let $M=\{s \in S$ : $\left.\mathcal{D}^{\prime}(s)=\emptyset\right\}$. The personal disks of the points in $M$ are hit by $p_{1}$ alone. The regions in $\mathcal{R}^{\prime}$ define an arrangement of pseudodisks (Lemma 2). In this arrangement we seek to find a point $q \in P$ of maximum depth. However, instead of finding a point with maximum depth, we find a point whose depth is within a constant factor of the maximum. We construct, in $O(n \log n)$ time, an approximate depth query data structure for the pseudodisks in $\mathcal{R}^{\prime}$ using Corollary 5.9 in [7] with a constant $\epsilon^{\prime} \leq 0.1$. This takes $O(n \log n)$ time. Then, for each point $p \in P$, we compute a value $\alpha(p)$ s.t. $0.9 \operatorname{depth}\left(p, \mathcal{R}^{\prime}\right) \leq \alpha(p) \leq \operatorname{depth}\left(p, \mathcal{R}^{\prime}\right)$ where $\operatorname{depth}\left(p, \mathcal{R}^{\prime}\right)$ denotes the depth of $p$ in the arrangement of regions in $\mathcal{R}^{\prime}$. This takes $O\left(\log ^{2} n\right)$ time per point and so the overall time taken is $O\left(n \log ^{2} n\right)$. We then take the point $p$ with the maximum $\alpha(p)$ as $q$. Observe that $|M|+\alpha(q)=\Theta\left(\rho\left(p_{1}\right)\right)$.

We first compute the set $S^{\prime}(q)=\left\{s \in S: q \in R^{\prime}(s)\right\}$. Note that $\left|S^{\prime}(q)\right|=\operatorname{depth}\left(q, \mathcal{R}^{\prime}\right) \geq \alpha(q)$. There are two cases to consider:

Case 1: $\left|S^{\prime}(q) \cup M\right|>8$. In this case, we set $p_{2}=q$ and let $Y=\left\{p_{1}, p_{2}\right\}$. Using Lemma 3, we can find a subset $X \subseteq S^{\prime}(q) \cup M$ so that $X=\Omega\left(\left|S^{\prime}(q) \cup M\right|\right)$ so that $\left(X,\left\{p_{1}, p_{2}\right\}\right)$ is a local improvement pair. Note that $|X|$ is $\Omega\left(\rho\left(p_{1}\right)\right)$. Thus in this case, we conclude that $p_{1}$ is useful and indeed we have found a local improvement that decreases the size of the current hitting set by $\Omega\left(\rho\left(p_{1}\right)\right)$.

Case 2: $\left|S^{\prime}(q) \cup M\right| \leq 8$. In this case $S^{\prime}(p) \leq\lfloor 8 / 0.9\rfloor=8$ for all $p \in P$. This means that $\rho\left(p_{1}\right)=O(1)$ and we just need to find one set $X$ of size 3 and a point $p_{2}$ so that $\left(X,\left\{p_{1}, p_{2}\right\}\right)$ is a local improvement pair. Using Lemma 5, we compute the set $S^{\prime}(p)$ for all $p \in P$ in $O(n \log n)$ expected time. For each $p_{2} \in P$, we need to check if there is any subset $X$ in $S^{\prime}\left(p_{2}\right) \cup M$ of size 3 so that $\left(X,\left\{p_{1}, p_{2}\right\}\right)$, is a local improvement pair. Since $\left|S^{\prime}\left(p_{2}\right) \cup M\right| \leq 8$, there are at most $\left(\begin{array}{l}8 \\ 3\end{array}\right)$ subsets $X \subseteq S^{\prime}\left(p_{2}\right) \cup M$ for which we need to check if $\left(X,\left\{p_{1}, p_{2}\right\}\right)$ is a local improvement pair. Thus there are $O(n)$ pairs of the form $\left(X,\left\{p_{1}, p_{2}\right\}\right)$, where $|X|=3$, that we need to check. For a particular pair of this form, we basically need to verify that all the disks in $\mathcal{D}$ whose intersection with $S$ is a subset of $X$ are hit by either $p_{1}$ or $p_{2}$. To make things simpler, we first remove from $\mathcal{D}$ all the disks that are hit by $p_{1}$ and obtain a set $\mathcal{D}^{\prime} \subseteq \mathcal{D}$. Now, we need to verify for all disks in $\mathcal{D}$ whose intersection with $X$ is a subset of $X$ that they are hit by $p_{2}$. All the $O(n)$ pairs can be checked in $O(n \log n)$ time as follows.

We construct a data structure that will help us do the checking for all the $O(n)$ pairs of the form $\left(X,\left\{p_{1}, p_{2}\right\}\right)$. We have already constructed a range reporting data structure on $S$ for disk ranges. Additionally, use a dictionary data structure (based on balanced binary trees) in which the keys are subsets of $S$ of size at most 3 and the value corresponding to a key $U$ is a list of disks $D \in \mathcal{D}^{\prime}$ s.t. $D \cap S=U$. We start with an empty dictionary. We 
then go over each disk $D \in \mathcal{D}^{\prime}$ one by one and if $N_{D} \leq 3$, we use the range reporting data structure to get $U=D \cap S$ in $O(\log n)$ time. We search the dictionary for $U$ and if it is found, we add $D$ to its list. If no entry is found, we create an entry for $U$ with a single element $d$ in its list. Note that since the number of $(\leq 3)$-sets that can be obtained from set of $n$ points by intersecting it with a set of disks is linear in the number of points [25], the number of distinct keys in the dictionary is $O(n)$. We go over each key $U$ and construct the region $R^{\prime}(U)$ by taking the intersection of all the disks in the list associated with $U$. Note that $R^{\prime}(U)$ can be constructed in $O(m \log m)$ time where $m$ is the size of the list associated with $U$ using Lemma 4. Since each disk is in the list of at most one $U$, the overall time is $O(n \log n)$. In the same amount of time, for each key $U$, we set up a data structure that allows us to check if a query point $q$ is in $R^{\prime}(U)$ using Lemma 4 . Now, to check if a pair $\left(X,\left\{p_{1}, p_{2}\right\}\right)$ is an improvement pair, we go over all subsets $U \subseteq X$ and check if $p_{2} \in R^{\prime}(U)$. The time spent for any pair is now $O(\log n)$. Therefore checking all the $O(n)$ pairs takes $O(n \log n)$ time.

If we find that none of the pairs we checked are local improvement pairs, then we can conclude that $p_{1}$ is not useful.

The following lemma shows how to find a profitable improvement. Let $\beta=\max \{\sqrt{t}, \epsilon t \cdot \mathrm{OPT} / n\}$.

Lemma 7. There exists $a k>0$ such that there are at least $\Omega(\beta / k)$ useful points $p \in P$ with $\rho(p) \geq k$.

Proof. By Lemma 1, there exists $\Omega(t)$ local improvement pairs $\left(X_{0}, Y_{0}\right), \ldots$ where the $X_{i}$ 's are disjoint subsets of $S$ but the $Y_{i}$ 's need not be disjoint. Each $X_{i}$ is of size 3 and each $Y_{i}$ is of size 2. For any pair of points $Y=\left\{p_{1}, p_{2}\right\} \subseteq P$, if $\left(X_{i}, Y\right)$ is a local improvement pair among the $\Omega(t)$ pairs, then we say that $X_{i}$ is a triple assigned to the pair $Y$. Define the weight of $Y$ as the number of triples assigned to it and denote it by $W(Y)$. The total weight of all pairs is then $\Omega(t)$.

Call a pair $Y$ to be of type $i$ if $2^{i-1} \leq W(Y)<2^{i}$, for $i=1, \ldots, O(\log t)$. If $W(Y)=0$ then we say that $Y$ is of type 0 . Since the total weight of all pairs is $\Omega(t)$, there must be some $j>0$ so that the total weight of the pairs of type $j$ is $\Omega\left(t / 2^{j}\right)$. Let $Q=\bigcup_{Y}\{Y \mid Y$ is of type $j\}$.

There are two lower bounds on the size of $Q$. First, since the total weight of the pairs of type $j$ is $\Omega\left(t / 2^{j}\right)$, and each pair has weight less than $2^{j}$, the number of pairs is $\Omega\left(t / 2^{2 j}\right)$, and hence $|Q|=\Omega\left(\sqrt{t} / 2^{j}\right)$. On the other hand, for any local improvement pair $\left(X_{i}, Y\right)$ where $Y$ is of type $j$, take any point $x \in X_{i}$. Since $\mathcal{D}(x)$ is non-empty, any disk $D \in \mathcal{D}(x)$ contains at least one point in $Y$. Therefore any such local improvement pair leads to an incidence between a point in $Q$ and a disk in $\mathcal{D}$. Note that since the $X_{i}$ 's are disjoint these are distinct incidences. Thus there are $\Omega\left(t / 2^{j}\right)$ incidences. Since by assumption no point in $P$, and therefore no point in $Q$, is in more than $n /(\epsilon \cdot$ OPT $)$ disks in $\mathcal{D}$, we have that $|Q|=\Omega\left(\epsilon t \cdot\right.$ OPT $\left./ 2^{j} n\right)$.

Therefore, $|Q|=\Omega\left(\max \left\{\epsilon t \cdot \mathrm{OPT} / 2^{j} n, \sqrt{t} / 2^{j}\right\}\right)=\Omega\left(\beta / 2^{j}\right)$. Observe that each $p \in Q$ is useful and $\rho(p) \geq$ $3 \cdot 2^{j}$. The lemma is therefore true for $k=2^{j}$.

We can now analyze the running time of our algorithm.

Running time: Preprocessing takes $O\left(n^{2}\right)$ time but this is dominated by the running time of Algorithm 1 . Consider a single iteration of the while loop in Algorithm 1. If we find some point $s \in S$ for which $\mathcal{D}(s)=\emptyset$, we drop $s$ from the current hitting set. This way we have improved the size of the hitting set at the cost of $O(n \log n)$ time. The total time spent on such improvements is at most $O($ OPT $n \log n)=O\left(n^{2} \log n\right)$. 
Otherwise, call a single iteration of the while loop lucky if the following is true:

$$
\exists i \text { such that the point } \pi_{i} \text { is useful and } \frac{i}{\rho\left(\pi_{i}\right)} \leq \frac{C n}{\beta}
$$

for some constant $C$.

Claim 2.2. Probability that any iteration of the whi le loop is lucky is at least $1 / 2$.

Proof. By Lemma 7, there exists a $k$ such that there are $\Omega(\beta / k)$ points, say the set $U$, with $\rho(p) \geq k$. Consider the smallest index $i$ s.t. $\pi_{i} \in U$. The expected value of $i$ is $O(n k / \beta)$. Therefore, with probability at least $\frac{1}{2}$, $i \leq C n k / \beta$ for some large enough $C$. Then,

$$
\frac{i}{\rho\left(\pi_{i}\right)} \leq \frac{C k n / \beta}{k}=\frac{C n}{\beta}
$$

Claim 2.3. For a lucky iteration of the while loop, let $\lambda$ be the reduction in size of the current hitting set, and $\sigma$ the time spent in this iteration. Then $\sigma / \lambda \leq C n^{2} \log ^{2} n / \beta$.

Proof. As we go over the points in random over, for the current point $\nu=\pi_{i}$, we estimate $\rho(\nu)$ which allows us to check if $i / \rho(\nu) \leq C n / \beta$. If so, assuming that the point $\nu$ is useful, we decrease the size of the current hitting set by $\Omega(\rho(\nu))$. If $i / \rho(\nu)>C n / \beta$ or we discover that $\nu$ is not useful we move to the next point in the random order. However, since the iteration of the while loop is lucky, we will find some point $\nu=\pi_{i}$ which is useful and for which $i / \rho(\nu) \leq C n / \beta$. For this point $\nu$, we find a local improvement involving $\nu$ of value $\Omega(\rho(\nu))$ and the current iteration of the while loop ends. The total time spent in this iteration is $\sigma=O\left(i \cdot n \log ^{2} n\right)$ since we have seen $i$ points so far and for each point we spend $O\left(n \log ^{2} n\right)$ time. The reduction in the size of the current hitting set is $\lambda=\Omega(\rho(\nu))$. Thus $\sigma / \lambda \leq \frac{i}{\rho(\nu)} \cdot n \log ^{2} n \leq C n^{2} \log ^{2} n / \beta$.

Since any iteration of the while loop is lucky with probaility at least 0.5 and we can assume that all the iterations are lucky. This does not change the running time by more than a factor of 2 .

Claim 2.4. The expected time taken to halve tis $O\left(n^{7 / 3} \log ^{2} n \epsilon^{-1 / 3}\right)$.

Proof. Claim 2.3 tells us that the amortized amount of time spent for the reducing the size of the current hitting set by 1 is $O\left(n^{2} \log ^{2} n / \beta\right)$. Since $\beta$ is an increasing function of $t$, this decreases with $t$. However, $t$ does not change by more than a factor of 2 until it is halved. So, the expected time for $t$ to be halved is $O\left(t / 2 \cdot n^{2} \log ^{2} n / \beta\right)$. Now, $t / \beta=\min \{\sqrt{t}, n /(\epsilon \cdot \mathrm{OPT})\}$. Since $t=O(\mathrm{OPT}), t / \beta=O(\min \{\sqrt{\mathrm{OPT}}, n /(\epsilon$. OPT $)\}=O\left((n / \epsilon)^{1 / 3}\right)$. Thus the expected time to halve $t$ is $O\left(n^{7 / 3} \log ^{2} n \epsilon^{-1 / 3}\right)$.

Since the initial value of $t$ is $O(\mathrm{OPT})$, there are $O(\log 1 / \epsilon)$ halving rounds until $t \leq \epsilon \cdot$ OPT. Thus, the expected running time of the Algorithm 1 is $O\left(n^{7 / 3} \log ^{2} n \epsilon^{-1 / 3} \log (1 / \epsilon)\right)$. Finally, since we need to run Algorithm 1 for $O(1 / \log (1+\epsilon))$ guesses for OPT, the overall running time is $O\left(n^{7 / 3} \log ^{2} n \epsilon^{-1 / 3} \log (1 / \epsilon) / \log (1+\epsilon)\right)$. For a fixed small value of $\epsilon$, this is $O\left(n^{7 / 3} \log ^{2} n\right)$. 


\section{Analysis of Quality of Local Search}

Let $R$ be a region in the plane. We say that a point $p \in \mathbb{R}^{2}$ hits $R$ if $p \in R$, and that a set of points $X$ hits a set of regions $\mathcal{R}$ if each region in $\mathcal{R}$ is hit by some point in $X$. We denote by $\mathcal{H}(P, \mathcal{R})$ the set system $(P,\{R \cap P: R \in \mathcal{R}\})$ induced by $P$ and $\mathcal{R}$. A hitting set for $\mathcal{H}(P, \mathcal{R})$ is a subset of $P$ which hits $\mathcal{R}$. A hitting set of the smallest cardinality is called the minimum hitting set and its size is denoted $\operatorname{OPT}(P, \mathcal{R}$ ) (or simply OPT when it is clear from the context). From now onwards, $P$ denotes a set of points and $\mathcal{D}$ denotes a set of (circular) disks in the plane. Our goal is to compute a hitting set for $\mathcal{H}(P, \mathcal{D})$ of a small size efficiently.

The analysis of the approximation factor achieved by a $(k, k-1)$-local search depends on the following theorem on planar bipartite graphs.

Theorem 1. $[12,26]$ Let $G=(R, B, E)$ be a bipartite planar graph on red and blue vertex sets $R$ and $B$, such that for every subset $B^{\prime} \subseteq B$ of size at most $k$, where $k$ is a large enough number, $\left|N_{G}\left(B^{\prime}\right)\right| \geq\left|B^{\prime}\right|$. Then, $|B| \leq(1+c / \sqrt{k})|R|$, where $c$ is a constant.

Here $N_{G}\left(B^{\prime}\right)$ denotes the set of neighbors of the vertices in $B^{\prime}$ in $G$. The proof of the above theorem, which relies on planar graph separators, requires $k$ to be quite large, thereby limiting the practical utility of the above theorem. A priori, it is not clear whether the theorems holds at all for small values of $k$. For instance, one can easily see that for $k=2$ there is no upper bound on $|B| /|R|$ (e.g., consider complete bipartite graph where $B$ is arbitrarily large and $|R|=2$ ). However, for $k=3$, we show a small bound of 8 on $|B| /|R|$, and then prove that it is, in fact, optimal.

Theorem 2. Let $G=(R, B, E)$ be a bipartite planar graph on red and blue vertex sets $R$ and $B$, such that for every subset $B^{\prime} \subseteq B$ of size at most $3,\left|N_{G}\left(B^{\prime}\right)\right| \geq\left|B^{\prime}\right|$. Then, $|B| \leq 8|R|$ and this bound is tight.

Proof. Let $n_{b}=|B|$ and $n_{r}=|R|$. Our goal is to prove that $n_{b} \leq 8 n_{r}$. Note that no vertex in $B$ can have degree 0 , otherwise the neighborhood of such a vertex is of size 0 , violating the conditions of the theorem. We make a new graph $G^{\prime}$ by adding edges in $G$ to all vertices of $B$ which have degree 1 in $G$. This can always be done while maintaining the planarity and bipartiteness of the graph as any such vertex $v$ must lie in a face which has at least two vertices of $R$, at least one of which is not adjacent to $v$. Thus in $G^{\prime}$ every vertex in $B$ has degree at least 2 . Let $n_{b_{2}}$ be the number of vertices of $B$ which have degree 2 and $n_{b_{\geq 3}}=n_{b}-n_{b_{2}}$ be the number of vertices of $B$ which have degree at least 3 in $G^{\prime}$. Since $G^{\prime}$ is planar and bipartite the number of edges in $G^{\prime} \leq 2\left(n_{b}+n_{r}\right)$. This implies that $2 n_{b_{2}}+3 n_{b_{\geq 3}} \leq 2 n_{b}+2 n_{r}$. Since $n_{b}=n_{b_{2}}+n_{b_{\geq 3}}$, we obtain $n_{b_{\geq 3}} \leq 2 n_{r}$.

We now show that $n_{b_{2}} \leq 6 n_{r}$. To do that we construct a graph $H$ with vertex set $R$ as follows: two vertices $r_{1} \in R$ and $r_{2} \in R$ are adjacent in $H$ iff there is at least one vertex $b \in B$ of degree 2 which is adjacent to both $r_{1}$ and $r_{2}$ in $G^{\prime}$. Note that $H$ is planar since the edge between $r_{1}$ and $r_{2}$ can be routed via one such $b$. Note that for the same pair $\left\{r_{1}, r_{2}\right\}$ there cannot be three vertices $b_{1}, b_{2}, b_{3} \in B$ of degree 2 each that are adjacent to both $r_{1}$ and $r_{2}$ since in that case the neighborhood of the set $\left\{b_{1}, b_{2}, b_{3}\right\}$ is of size 2 violating the conditions of the theorem. Therefore, each vertex $b \in B$ of degree 2 corresponds to an edge in $H$ and each edge has at most two vertices in $B$ that correspond to it. Since the number of edges in $H$ is at most $3|R|=3 n_{r}$, we conclude that $n_{b_{2}} \leq 6 n_{r}$. Thus $n_{b}=n_{b_{2}}+n_{b_{\geq 3}} \leq 6 n_{r}+2 n_{r}=8 n_{r}$. 
We now show that the bound given above is tight. However, that still leaves open the possibility that, by exploiting other properties of disks, a $(3,2)$-local search could give a better approximation for the problem of computing minimum hitting sets for disks in the plane. The following theorem rules this out.

Theorem 3. For any $\delta>0$, there exists an integer $n_{0}$ such that one can construct a set $\mathcal{D}$ of disks in the plane, a set of points $P,|P| \geq n_{0}$, and a subset $B \subseteq P$ s.t. $\left.i\right) B$ is a hitting set for $\mathcal{H}(P, \mathcal{D})$, ii $)|B| \geq(8-\delta)$ OPT and iii) there are no subsets $X \subseteq B$ and $Y \subseteq P \backslash B,|Y|<|X| \leq 3$, s.t. $(B \backslash X) \cup Y$ is a hitting set for $\mathcal{H}(P, \mathcal{D})$.

Proof. We first construct a bipartite graph $G=(R, B, E)$ that satisfies the conditions of Theorem 2 and $|B| \geq(8-\delta)|R|$. Let $L$ be the triangular lattice, and take a large equilateral triangle $\Delta$ aligned with the edges of $L$ (so that $L \cap \Delta$ triangulates $\Delta$ ) and containing many faces of the lattice. Then replace each face of the lattice by the block of the type shown in Figure 2(a). The corner vertices (unshaded) of the block map to the corner vertices of the face, while the other vertices (shaded) in the block lie in the interior of the face. Let $R$ be the set of vertices of $L$ lying in $\Delta$ and let $B$ be the set of vertices lying in the interior of the faces in $L \cap \Delta$. The blocks together define a bipartite graph (see Figure 2(b) for a small example with four blocks put together; note that the edges of $L$ are drawn in bold). The dotted edges and the edges of the lattice $L$ are not part of the graph. Note that each face in $L \cap \Delta$ contains four points of $B$, and if $\Delta$ is large enough, the number of faces of $L$ in $\Delta$ is nearly twice the number of vertices of $L$ in $\Delta$. The size of $\Delta$ can be chosen, depending on $\delta$, such that the number of faces of $L$ is at least $(2-\delta / 4)$ times the number of vertices of $L$. Thus we get that $|B| \geq(8-\delta)|R|$. It can be verified by inspection that there is no subset of $B$ of size at most 3 with a smaller neighborhood. This shows that the bound in Theorem 2 is tight within additive constants.

Now, we extend $G$ to a triangulation by including the dotted edges in the blocks. Note that there are some dotted edges going between blocks. We also put an additional vertex in the outer face and connect it to all vertices in the outer face of $G$ (i.e. we stellate the outer face). The resulting graph, call it $\Xi$, is triangulated (i.e., each face is of size 3) and furthermore it is 4-connected since, as can be verified by inspection, there is no separating triangle (a non-facial cycle of length 3). By a theorem of Dillencourt and Smith (Theorem 3.5 in [14]), there exists an embedding of $\Xi$ in the plane so that $\Xi$ is the Delaunay triangulation of its vertices. Abusing notation, we refer to the embedding as $\Xi$ and we refer to the embedding of a vertex $v$ in $\Xi$ as $v$.

Let $R$ and $B$ thus be the two sets of points. We set $P=R \cup B$, and construct $\mathcal{D}$ by taking for each edge $e$ in $G$ a disk that contains exactly the two end points of $e$ among all the vertices in $\Xi$. This is possible because $\Xi$ is now a Delaunay triangulation of the points in $P$. By construction, each disk in $\mathcal{D}$ contains exactly one point from each of the sets $R$ and $B$ and thus both the sets are hitting sets for $\mathcal{H}(P, \mathcal{D})$. Since OpT is the size of the smallest hitting set, OPT $\leq|R|$ and therefore $|B| \geq(8-\delta)$ OPT. Consider a local improvement step where we seek to decrease the size of the hitting set $B$ by removing some subset $X \subseteq B$ of size at most 3 and adding a smaller set $Y$ outside $B$ (i.e., $Y \subseteq R$ ) so that $(B \backslash X) \cup Y$ is a hitting set for $\mathcal{D}$. Let $x$ be one of the points in $X$. Observe that then all neighbors of $x$ in $G$ must be in $Y$ since for each neighbor $y$ of $x$, there is a disk in $\mathcal{D}$ which contains only the two points $x$ and $y$ among all the points in $R \cup B$. This means that $|Y| \geq\left|N_{G}(X)\right|$. Since for any $X$ of size at most $3,\left|N_{G}(X)\right| \geq|X|$, we have that $|Y| \geq|X|$ implying that such a local improvement is not possible.

As mentioned before, a (4,3)-local search gives a 5 approximation. 


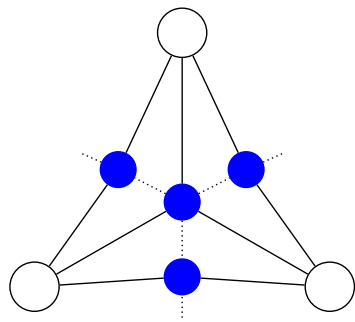

(a)

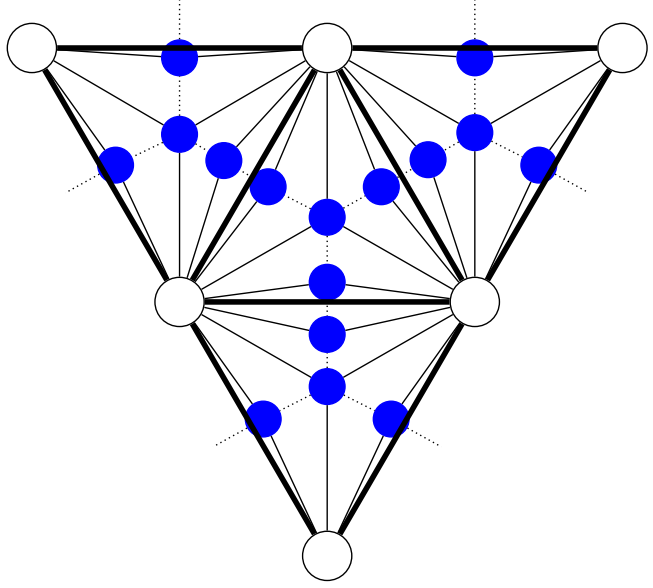

(b)

Figure 2: Unshaded vertices correspond to red and shaded to blue vertices. The dotted lines show a triangulation. We tile the triangles in (a) as shown in (b). The ratio of shaded to unshaded vertices goes to 8 as size of the tiling is increased. Connecting the vertices at the boundary of the tiling to a new vertex gives a 4-connected graph.

Theorem 4. Let $G=(R, B, E)$ be a bipartite planar graph on red and blue vertex sets $R$ and $B$, such that for every subset $B^{\prime} \subseteq B$ of size at most $4,\left|N_{G}\left(B^{\prime}\right)\right| \geq\left|B^{\prime}\right|$. Then, $|B| \leq 5|R|$. Furthermore, there are such graphs with $|B| \geq 4|R|$.

Proof. Let $n_{b}=|B|$ and $n_{r}=|R|$. As in the proof of Theorem 2, we construct a graph $G^{\prime}$ such that every vertex of $B$ has degree at least 2 . As before we can show that $n_{b_{\geq 3}} \leq 2 n_{r}$.

We now show that $n_{b_{2}} \leq 3 n_{r}$. We construct the graph $H$ as before. Since the vertex set of $H$ is a subset of $R$, the number of vertices in $H$ is at most $n_{r}$. Each edge in $H$ has either one or two blue vertices corresponding to it. We assign to each edge a weight equal to the number of blue vertices corresponding to it. The total weight of all edges in $H$ gives $n_{b_{2}}$. Recall that $H$ is planar and therefore we can complete it to a triangulation $T$ by adding additional edges of weight 0 so that the total weight of the edges is unchanged. Consider any (triangular) face $t$ of this triangulation. The total weight of the edges of this face cannot be 4 or more since that corresponds to the existence of four or more blue vertices with a neighborhood of size at most 3 (the vertices of $t$ ), violating the conditions of the theorem.

We distribute the weight of the edges of $T$ to the faces of $T$ as follows: each edge distributes its weight equally among the two faces adjacent to it. Since the edges surrounding any face have total weight at most 3 , each face gets a weight of at most 1.5. Since the number of faces in the triangulation is at most twice the number of vertices $\left(2 n_{r}\right)$, we obtain that the total weight of all the faces is at most $3 n_{r}$. In other words, $b_{n_{2}} \leq 3 n_{r}$. Thus $b=n_{b_{\geq 3}}+n_{b_{2}} \leq 5 n_{r}$.

For the lower-bound construction, see Figure 3. Tiling the square into a $k \times k$ grid gives $4 k^{2}$ vertices in $B$, 

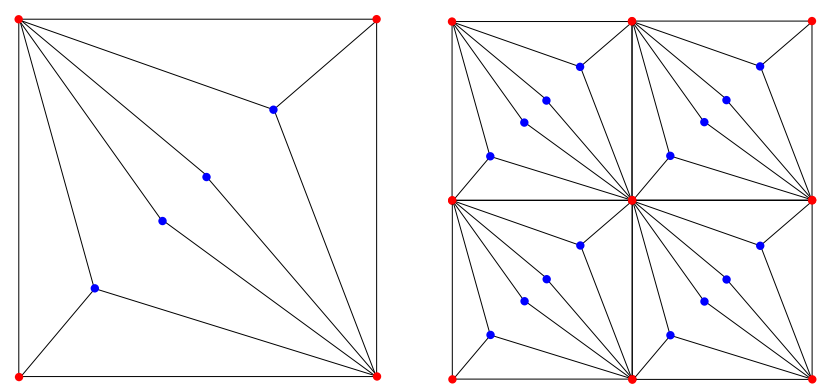

Figure 3: Lower-bound construction for Theorem 4.

while $R$ has $k^{2}+k+1$ vertices. It can verified that this graph satisfies the conditions of the theorem. 


\section{References}

[1] Rashmisnata Acharyya, Manjanna Basappa, and Gautam K. Das. Unit disk cover problem in 2d. In Computational Science and Its Applications - ICCSA 2013 - 13th International Conference, Ho Chi Minh City, Vietnam, June 24-27, 2013, Proceedings, Part II, pages 73-85, 2013.

[2] Peyman Afshani and Timothy M. Chan. Optimal halfspace range reporting in three dimensions. In SODA, pages 180-186, 2009.

[3] Pankaj K. Agarwal, Esther Ezra, and Micha Sharir. Near-linear approximation algorithms for geometric hitting sets. Algorithmica, 63(1-2):1-25, 2012.

[4] Pankaj K. Agarwal, János Pach, and Micha Sharir. State of the Union (of Geometric Objects): A Review, 2007.

[5] Pankaj K. Agarwal and Jiangwei Pan. Near-linear algorithms for geometric hitting sets and set covers. In Symposium on Computational Geometry, page 271, 2014.

[6] Christoph Ambühl, Thomas Erlebach, Matús Mihalák, and Marc Nunkesser. Constant-factor approximation for minimum-weight (connected) dominating sets in unit disk graphs. In APPROX-RANDOM, pages 3-14, 2006.

[7] Boris Aronov and Sariel Har-Peled. On approximating the depth and related problems. SIAM J. Comput., 38(3):899921, 2008.

[8] Hervé Brönnimann and Michael T. Goodrich. Almost optimal set covers in finite vc-dimension. Discrete \& Computational Geometry, 14(4):463-479, 1995.

[9] Norbert Bus, Shashwat Garg, Nabil H. Mustafa, and Saurabh Ray. Improved local search for geometric hitting set. In STACS, 2015.

[10] Gruia Călinescu, Ion I. Mandoiu, Peng-Jun Wan, and Alexander Zelikovsky. Selecting forwarding neighbors in wireless ad hoc networks. MONET, 9(2):101-111, 2004.

[11] Paz Carmi, Matthew J. Katz, and Nissan Lev-Tov. Covering points by unit disks of fixed location. In ISAAC, pages 644-655, 2007.

[12] Timothy M. Chan and Sariel Har-Peled. Approximation algorithms for maximum independent set of pseudo-disks. In Symposium on Computational Geometry, pages 333-340, 2009.

[13] Francisco Claude, Gautam K. Das, Reza Dorrigiv, Stephane Durocher, Robert Fraser, Alejandro López-Ortiz, Bradford G. Nickerson, and Alejandro Salinger. An improved line-separable algorithm for discrete unit disk cover. Discrete Math., Alg. and Appl., 2(1):77-88, 2010.

[14] Michael B. Dillencourt and Warren D. Smith. Graph-theoretical conditions for inscribability and delaunay realizability. Discrete Mathematics, 161(13):63 - 77, 1996.

[15] G. Even, D. Rawitz, and S. Shahar. Hitting sets when the VC-dimension is small. Inf. Process. Lett., 95:358-362, 2005.

[16] Eti Ezra, Dan Halperin, and Micha Sharir. Speeding up the incremental construction of the union of geometric objects in practice. Comput. Geom., 27(1):63-85, 2004. 
[17] Robert Fraser. Algorithms for Geometric Covering and Piercing Problems. PhD thesis, University of Waterloo, 2012.

[18] Shashidhara K. Ganjugunte. Geometric Hitting Sets and Their Variants. PhD thesis, Duke University, 2011.

[19] Alejandro Lpez-Ortiz Gautam K. Das, Robert Fraser and Bradford G. Nickerson. On the discrete unit disk cover problem. International Journal on Computational Geometry and Applications, 22(5):407-419, 2012.

[20] Matt Gibson and Imran A. Pirwani. Algorithms for dominating set in disk graphs: Breaking the $\log n$ barrier (extended abstract). In Algorithms - ESA 2010, 18th Annual European Symposium, Liverpool, UK, September 6-8, 2010. Proceedings, Part I, pages 243-254, 2010.

[21] D. S. Hochbaum and W. Maass. Fast approximation algorithms for a nonconvex covering problem. J. Algorithms, 8(3):305-323, 1987.

[22] Dorit S. Hochbaum. Approximation algorithms for the set covering and vertex cover problems. SIAM J. Comput., 11(3):555-556, 1982.

[23] Erik Krohn, Matt Gibson, Gaurav Kanade, and Kasturi R. Varadarajan. Guarding terrains via local search. JoCG, 5(1):168-178, 2014.

[24] Dániel Marx. Parameterized complexity of independence and domination on geometric graphs. In Parameterized and Exact Computation, Second International Workshop, IWPEC 2006, Zürich, Switzerland, September 13-15, 2006, Proceedings, pages 154-165, 2006.

[25] J. Matousek. Lectures in Discrete Geometry. Springer-Verlag, New York, NY, 2002.

[26] Nabil H. Mustafa and Saurabh Ray. Improved results on geometric hitting set problems. Discrete \& Computational Geometry, 44(4):883-895, 2010.

[27] Thomas Ottmann, Peter Widmayer, and Derick Wood. A fast algorithm for the boolean masking problem. pages 249-268, 1985.

[28] János Pach and Micha Sharir. Combinatorial Geometry with Algorithmic Applications the Alcala Lectures, 2006.

[29] E. Pyrga and S. Ray. New existence proofs for epsilon-nets. In Proceedings of Symposium on Computational Geometry, pages 199-207, 2008.

[30] R. Raz and M. Safra. A sub-constant error-probability low-degree test, and a sub-constant error-probability PCP characterization of NP. In Proceedings of STOC, pages 475-484, 1997. 